
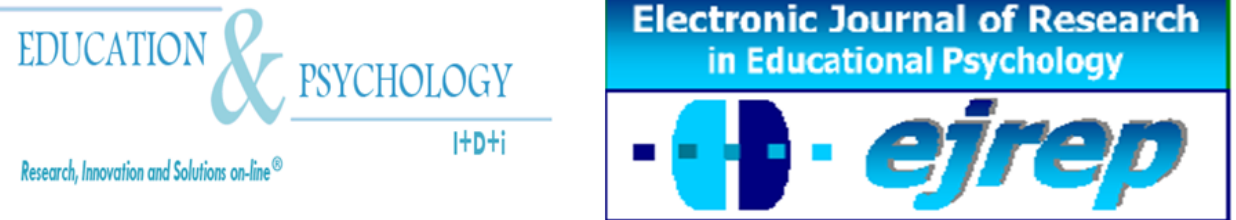

Editorial EOS

\title{
Relación entre optimismo, creatividad y síntomas psicopatológicos, en estudiantes universitarios
}

\begin{abstract}
Óscar Sánchez Hernández ${ }^{1}$, Ramón Martín-Brufau ${ }^{2}$, Francisco Xavier Méndez Carrillo ${ }^{3}$, Francisco Javier Corbalán Berna ${ }^{4}$, Rosa María Limiñana Gras ${ }^{5}$
\end{abstract}

Departamento de Personalidad, Evaluación y Tratamiento Psicológicos. Facultad de Psicología. Universidad de Murcia.

España

Correspondencia: Óscar Sánchez Hernández. Facultad de Psicología. Campus Universitario de Espinardo (Murcia). CP: 30100. España. E-mail: oscarsh@um.es

(C) Education \& Psychology I+D+i and Editorial EOS (Spain) 


\section{Resumen}

Introducción. En este estudio se analiza el efecto protector de las variables de optimismo disposicional y creatividad respecto a una medida de psicopatología o sufrimiento psicológico.

Método. Un total de 113 estudiantes universitarios de un curso de promoción educativa participaron en la investigación. Durante la realización de este curso se administraron medidas de creatividad (CREA), optimismo (LOT-R) y síntomas psicopatológicos (SCL-90). Se efectuaron análisis correlacionales y de regresión utilizando el optimismo o la creatividad como variables predictoras sobre los síntomas psicopatológicos. Para completar los análisis se efectuaron ANOVAS entre los niveles de creatividad divididos en tres grupos baja, media y alta creatividad.

Resultados. Se encontraron fuertes correlaciones negativas y estadísticamente significativas del optimismo disposicional respecto de los síntomas psicopatológicos. Los síntomas psicopatológicos medidos a través del SCL-90 eran explicados por la variable optimismo disposicional, principalmente con respecto a las dimensiones de depresión y dificultades interpersonales. Sin embargo, no se encontraron correlaciones significativas entre la creatividad y la medida de psicopatología. Se encontraron diferencias en síntomas psicopatológicos en función de los tres grupos de creatividad, siendo los participantes con un nivel intermedio de creatividad los que presentan menores sintomas psicopatológicos.

Discusión y Conclusiones. A la luz de los resultados de este estudio, se confirmó el efecto protector del optimismo frente a la psicopatología encontrado en otros estudios y se proponen propuestas concretas de aplicación en programas de promoción del bienestar psicológico. Se barajan diversas propuestas para explicar la relación hallada entre la creatividad y la psicopatología.

Palabras Clave: Optimismo, creatividad, salud mental, psicopatología 


\begin{abstract}
Introduction. This study examines the protective effects of variables of dispositional optimism and creativity with respect to measurements of psychopathology or psychological distress.

Method. A total of 113 university students from different degree programs participated in the research. Measures of creativity (CREA), optimism (LOT-R) and psychopathological symptoms (SCL-90) were administered during their course on public speaking. Correlational and regression analyses were carried out using optimism and creativity as predictors for psychopathological symptoms. In order to round out the analysis, ANOVAS were carried out between three levels of creativity, with groups fomed according to low, medium and high creativity.
\end{abstract}

Results. Strong negative correlations, statistically significant, were found between dispositional optimism and psychopathological symptoms. The psychopathological symptoms measured by the SCL-90 were explained by the variable of dispositional optimism, primarily in its dimensions of depression and interpersonal difficulties. However, no significant correlational relationship was found between creativity and the measure of psychopathology. Differences in psychopathological symptoms, however, were found according to the three groups of creativity; participants with a medium level of creativity showed the least psychopatological symptoms.

Discussion and Conclusions. In the light of the results of this study, the protective effect of optimism against psychopathology, as found in other studies, was confirmed, and we offer specific proposals to be applied in psychological well-being programs. Various possibilities are suggested to explain the relationship found between creativity and psychopathology.

Keywords: Optimism, creativity, mental health, psychopathology

Received: 05/11/10 Initial Acceptance: 05/17/10 Final Acceptance: 07/30/10 
Oscar Sánchez et al. 


\section{Introducción}

En el libro titulado "La personalidad creadora", el famoso psicólogo humanista, Abraham H. Maslow (2005), cita textualmente "El sentido común significa vivir en el mundo tal cual es, pero las personas creativas son aquéllas que no aceptan el mundo tal cual es, sino que quieren construir otro mundo" (p. 122). La creatividad requiere "salirse de las vías normales" para encontrar ideas, problemas o soluciones que normalmente no están presentes de forma habitual, sino que solo es real en la mente del que juega e inventa realidades alternativas para nuevos problemas y retos que se van dando. El optimismo, por su parte, es capaz de ver, considerar y perseguir realidades que no tienen porque estar pasando en este momento sino que la persona espera que las cosas mejoren o que seguirán siendo buenas en el futuro, contribuyendo de forma activa a esa realidad alternativa y positiva, que va construyendo con sus acciones, pensamientos y sentimientos en el momento presente para la que será necesaria persistencia y tenacidad, lo que le ayudará a lograr las metas propuestas (Carr, 2007 ; Carver y Scheier, 1998).

Tanto el optimismo como la creatividad pueden ser factores que faciliten adaptarse a los nuevos contextos, caracterizados por rápidos cambios. Por otra parte, aunque sabemos que el optimismo es productor de emociones positivas (Vera, 2008), incluso en situaciones adversas, la creatividad podría caracterizarse por cierta insatisfacción producida por ese inconformismo a lo que ahora es, que incite a esa búsqueda de realidades alternativas a las presentes.

La promoción de la salud mental y prevención de trastornos psicológicos ha experimentado un incremento de la investigación en los últimos años (Horowitz y Garber, 2006). Seligman y Csikszentmihalyi (2000) señalan que el progreso mayor de la prevención procede de una perspectiva centrada en el desarrollo sistemático de competencias.

\section{Optimismo y psicopatología}

Avia y Vázquez (1998) indican que el optimismo, además de ser requisito para la supervivencia, es condición indispensable para una vida plenamente humana, una de las mejores armas para adaptarse al medio y transformarlo, un mecanismo que permite que las personas sean capaces de superar el infortunio, de construir y soñar el futuro. Según Vera (2008) exis- 
ten dos perspectivas teóricas diferentes pero no opuestas a la hora de comprender el optimismo:

1. Estilo explicativo (Abramson, Seligman y Teasdale, 1978). El modelo reformulado de indefensión aprendida se basa en la teoría atribucional y sostiene que las expectativas sobre acontecimientos futuros están estrechamente relacionadas con la explicación de los acontecimientos pasados. El estilo explicativo es la forma característica de la persona de interpretar sus experiencias. Comprende tres dimensiones: a) personalización, distingue si la causa del suceso radica en la persona o en un factor externo (atribución interna o externa); b) duración, se refiere al grado en que la causa es estable en el tiempo y propende a repetirse (atribución permanente o temporal); amplitud, examina si la causa afecta o no a otras áreas de la vida (atribución global o específica). La persona optimista tiende a explicar los eventos negativos mediante atribuciones externas, temporales y específicas mientras que la pesimista realiza atribuciones internas, permanentes y globales. Por el contrario, los acontecimientos positivos se explican de forma inversa.

Los beneficios del estilo explicativo optimista son numerosos. Se asocia a índices más bajos de enfermedad, de depresión y de suicidio, y a niveles más elevados de rendimiento académico y deportivo, adaptación profesional y calidad de vida familiar (Gillham, 2000; Sánchez y Méndez, 2009b; Seligman, 1998; Seligman, Reivich, Jaycox, y Gillham, 2005). Las investigaciones realizadas en un contexto educativo durante cinco años con más de 500 niños participantes en el Estudio Longitudinal de la Depresión Infantil muestran que la explicación optimista amortigua el impacto perjudicial de la adversidad y protege de la depresión, mientras que el estilo pesimista aumenta el riesgo (Nolen-Hoeksema, Girgus y Seligman, 1992; Seligman et al., 1984).

El optimismo se relaciona con la capacidad para demorar las gratificaciones y renunciar a los beneficios a corto plazo a cambio de objetivos a largo plazo más valiosos, probablemente porque la persona considera alcanzables dichos objetivos (Carr, 2007). Sánchez, Méndez y Garber (2009) en un estudio con 87 adolescentes en el contexto educativo acerca de la motivación para participar en un programa de promoción del bienestar psicológico, encuentran que la curiosidad acerca del cambio positivo se relaciona de forma positiva con el estilo explicativo optimista y de forma negativa con la sintomatología ansiosa de los participantes, por lo que una visión más optimista del cambio facilita la curiosidad y motivación 
para introducir cambios positivos en la vida (aprender comportamientos saludables que aumenten el bienestar físico y psicológico de los estudiantes, marcarse y seguir un plan de acción para conseguir metas personales, etc). También se considera un factor protector del consumo de sustancias en situaciones estresantes (Torres Jiménez, Robert, Tejero, Boget y Pérez de los Cobos, 2006).

Dentro de esta concepción del optimismo como estilo explicativo, tenemos que resaltar que los autores no se limitan a realizar un modelo explicativo, junto a cuestionarios para medir este constructo, sino que proponen el Programa de Resistencia a la Adversidad (Penn Resiliency Program, PRP), de la Universidad de Pensilvania (Gillham, Jaycox, Reivich, Seligman y Silver, 1990) con la finalidad de fomentar el optimismo en los participantes con los beneficios que conlleva. Existen al menos 14 estudios con asignación al azar de los participantes y grupo de control, realizados por los creadores del PRP y por otros equipos de investigación, en los que han participado más de 2.000 niños y adolescentes de 8 a 15 años (Abela y Hankin, 2007). En conjunto los resultados revelan que el programa reduce de forma duradera los síntomas de ansiedad y depresión. Gillham, Hamilton, Freres, Patton y Gallop (2006) encontraron que el PRP previene trastornos del estado de ánimo, de ansiedad y de adaptación (combinados), en un seguimiento de dos años a niños con elevados niveles de sintomatología. También se ha hallado que el efecto preventivo sobre el comportamiento disruptivo se mantiene 2 - 3 años después de finalizar el programa (Cutuli, 2004; Cutuli, Chaplin, Gillham, Reivich y Seligman, 2006).

López, Kasanzew y López (2007) en una muestra de 60 pacientes de baja y media gravedad psicopatológica, asignaron al azar al grupo control (psicoterapia cognitiva clásica) o al grupo experimental (psicoterapia cognitiva clásica más técnicas de fomento del optimismo) logrando una optimización y potenciación de los efectos de la psicoterapia cognitiva clásica en las primeras cinco sesiones al aumentar la visión optimista, utilizando las técnicas del fomento de optimismo de Seligman (2005) y disminuir la intensidad de los síntomas psicopatológicos en los pacientes, utilizando como medida de psicopatología la escala SCL-90-R (Casullo, 2004). Los resultados mostraron una disminución significativa en variables como somatizaciones, ansiedad fóbica y hostilidad a favor del grupo experimental en comparación con el grupo control. Por lo tanto, este plan para aumentar el optimismo parece producir una optimización de la psicoterapia cognitiva al disminuir la intensidad de los síntomas en los pacientes. 
Sánchez y Méndez (2009a) realizaron un estudio piloto de un programa cognitivoconductual inspirado en el PRP, con el objetivo de fomentar el optimismo en 25 estudiantes de primaria, encontrando que en el grupo experimental se redujo significativamente la sintomatología depresiva en el postest, obteniéndose un tamaño del efecto medio-bajo en consonancia con los resultados de la investigación en este campo.

Resaltar, dentro del contexto educativo, el papel decisivo de padres, profesores, entrenadores y de cualquier agente educativo en general, en la formación del estilo explicativo de niños y adolescentes, tanto por la forma de explicarse estos agentes lo que les sucede en sus vidas a ellos mismos, y más importante, cómo explican con sus comentarios lo que les ocurre a sus educandos, lo que contribuirá de forma decisiva en el desarrollo del optimismo o pesimismo de los estudiantes, con las consiguientes consecuencias para su bienestar físico y psicológico, así como en el resto de áreas de sus vidas (Seligman, 2005).

2. Característica disposicional (Scheier y Carver, 1985). Estos autores definieron el optimismo disposicional como una expectativa o creencia estable, generalizada, de que en la vida ocurrirán cosas positivas (Scheier y Carver, 1985, 1987) por lo que el optimismo sería la tendencia a esperar que en el futuro ocurran resultados favorables, en tanto que el pesimismo correspondería a la expectativa de que en el futuro ocurran eventos negativos (Avia y Vásquez, 1998; Otero et al., 1998). El antecedente teórico de este concepto vendría del modelo de autorregulación de conducta de Carver y Scheier (1981) según el cual, cuando surgen dificultades las expectativas favorables incrementan los esfuerzos de las personas para alcanzar los objetivos y las expectativas desfavorables reducen tales esfuerzos, a veces hasta el punto de desentenderse totalmente de la tarea (Carver y Scheier, 1998).

El gran interés que ha generado el optimismo disposicional ha sido debido a su gran poder predictivo, tanto de bienestar psicológico y salud física, como de las estrategias de afrontamiento utilizadas para superar las distintas situaciones que se van planteando en el transcurso de la vida (Sanjuán y Magallanes, 2006). Diversos estudios han encontrado que los optimistas experimentan menos emociones negativas, como estado de ánimo negativo o sintomatología depresiva, ansiedad u hostilidad cuando se enfrentan con situaciones estresantes (Sanjuán y Magallanes, 2006). Se ha comprobado que los sujetos optimistas afrontan mejor los problemas de la vida, sufren menos estrés y por lo tanto enfermarán menos (Ortiz, Ramos y Vera-Villarroel, 2003). Específicamente en relación con las estrategias de afrontamiento se 
ha encontrado que los sujetos optimistas tienen más estrategias de afrontamiento centradas en el problema que los pesimistas, valoran de forma más positiva las situaciones estresantes y buscan más apoyo social. En cambio, los sujetos pesimistas tienden a negar, a rendirse y a no comprometerse con las metas, enfocándose en los sentimientos negativos (Carver y Gaines, 1987; Scheier, Weintraub y Carver, 1986).

\section{Creatividad y psicopatología.}

Tradicionalmente, la creatividad se ha relacionado con la psicopatología (Andreasen, 1996). En un principio Lombroso (1891) relacionó los individuos más creativos con niveles mayores de psicopatología. Sin embargo, esta posición ha resultado muy controvertida, hasta el punto que podría decirse que existen dos líneas separadas de investigación respecto a la psicopatología y la creatividad (Alonso, 2000) que han aportado pruebas a favor y en contra de esta hipótesis: las que establecen una relación positiva (Carlsson, 2002; Clapham, 2001) y las que establecen una relación negativa (Mikulincer, Kedem y Paz, 1990) entre éstas.

Varios estudios han mostrado que existe relación entre la capacidad creativa y desórdenes mentales, generalmente relacionados con el espectro esquizofrénico: laxitud asociativa, mayor amplitud atencional y la habilidad de conectar nueva información de forma poco convencional (Richards, 2001; Woody y Claridge, 1977). Se han encontrado datos que evidencian una asociación entre la creatividad y los rasgos esquizotípicos por compartir características cognitivas y emocionales similares (Joscelyn et al., 2004). Esto se relaciona con el hecho de que los familiares de pacientes esquizofrénicos presenten mayores niveles de creatividad, que los pacientes bipolares cuenten un mayor número de logros creativos (Claridge y Blakey, 2009) y de que mayores niveles de rasgos esquizotípicos de personalidad se relacionen con mayor número de estrategias de solución de problemas aunque a costa de más tiempo para resolverlos (Stoneham y Coughtrey, 2009). Algunos autores han propuesto que los rasgos esquizotípicos facilitan el procesamiento divergente, mientras que la alteración emocional en forma de manía provee del impulso y el humor necesario para la producción creativa (Nettle, 2002). Quizá por eso se hallan encontrado datos de que la creatividad no solo no está asociada con la psicopatología sino que se encuentra que un predominio de emociones positivas beneficiaría a la creatividad (Lyubomirsky, King y Diener, 2005) tal vez relacionado con estados hipmaníacos (Nettle, 2006). 
Este hecho podría entrar en la línea de lo que algunos autores han sugerido como adicción a la novedad en las personas con alta creatividad (Galang, 2010). Según estas propuestas, los individuos más creativos presentarían una alta motivación para la novedad, lo que tal como sugieren los autores podría ser una forma de auto-regulación para paliar los estados derivados de una baja activación cortical mediante una capacidad de generar ideas novedosas. Esta adicción a la novedad debería estar al menos en parte relacionada con las bases de la adicción, según la cual las personas con menor número de receptores de D2 necesitaría mayores niveles de estimulación para sentir los efectos recompensantes de las actividades habituales (Volkow, Fowler, Wang, y Swanson, 2004). Esta propuesta se ve respaldada por estudios que confirman que los individuos que puntúan alto en producción divergente presentan menores receptores D2 lo que podría aportar luz a la relación entre creatividad y la psicopatología del espectro esquizofrénico asociada a ésta (De Manzano, Cervenka, Karabanov, Farde y Ullèn, 2010).

Aunque la creatividad puede presentar formas características de procesar la información, lo que parece aceptado es que la alteración emocional no es un prerrequisito para la creatividad en el sentido de exisitir una relación causal entre ellas (Glover, Ronning y Reinolds, 1989). Algunos hallazgos prometedores subrayan la importancia de la inteligencia como umbral a partir del cual la creatividad se ve favorecida. Esta propuesta no ha sido confirmada por diferentes estudios (Kim, 2005; Preckel, Holling y Wiese, 2006). Sin embargo, recientes propuestas subrayan la importancia de la inteligencia medida a través de la memoria de trabajo como una capacidad directamente relacionada con la producción divergente (Vandervert, 2007). Así, una distinción entre los individuos con alta creatividad reside en ser capaces de seleccionar aquellas asociaciones más plausibles y más prometedoras (Galang, 2010), algo que los individuos con sintomatología esquizofrénica no pueden llegar a realizar correctamente dando lugar a sobreinlusión e ideaciones poco productivas debido a déficits en el control de la inhibición cognitiva (Lubow y Gewirtz, 1995). Así, la creatividad podría estar asociada a la psicopatología por su relación con variables de personalidad que podrían predisponer a ella, más que por su relación directa con la alteración emocional (Chavez-Eakle, Lara y Cruz-Fuentes, 2006). Como reconoce Behrens (1975), la diferencia entre el creativo y el lunático sería la misma que entre Cervantes y Don Quijote: uno construye metáforas, el otro las cree reales. Por lo tanto, ¿Supone la creatividad un factor protector ante la psicopatología? ¿Supone un riesgo por su asociación con formas alteradas de pensamiento, con estilos cognitivos que puedan producir también síntomas psicopatológicos? 
En un estudio metaanalítico relacionando emociones positivas y negativas con la creatividad se dedujo que la creatividad estaba relacionada de forma compleja con las emociones positivas y con las negativas (Baas, De Drew y Bernard, 2008). Sugieren que quizá no se trate de establecer qué tipo de valencia emocional, negativa o positiva generan mejores efectos sobre la creatividad, sino su interacción con los niveles de activación y los efectos cognitivos relacionados con la creatividad (flexibilidad cognitiva, fluidez, originalidad, etc...). En concreto, en este metaanálisis, también se encontró un aumento del producto creativo en relación a emociones negativas como ansiedad y miedo por la vía de persistir en un objetivo y focalizar la atención en él (Baas, De Drew y Bernard, 2008), características necesarias para el desarrollo de un producto creativo. En definitiva este metaanálisis encuentra que la variable tono hedónico y el nivel de activación interactúan: ninguno es suficiente para explicar los efectos de las emociones sobre la creatividad. Por lo tanto, en algunas situaciones las emociones negativas también podrían favorecer la creatividad. En ocasiones, dada una capacidad creativa, el producto o el rendimiento creativo puede verse fomentado o favorecido por emociones tanto positivas como negativas (que pueden conducir a más síntomas psicopatológicos). Por lo tanto, se necesitaría más investigación para tratar de comprender la relación entre psicopatología y creatividad.

\section{Objetivos e hipótesis}

1. Evaluar la hipótesis del optimismo como factor protector de psicopatología en consonancia con los datos aportados en otros estudios, por lo que el optimismo se relacionará de forma negativa con los síntomas psicopatológicos. Aportar esta evidencia relacionando la medida del optimismo disposicional, LOT-R (Scheier, Carver y Bridges, 1994) con la medida de psicopatología SCL-90 (Derogatis, Lipman y Covi, 1973), ya que no se encontró otras investigaciones que lo hayan estudiado.

2. Comprobar si, en población universitaria estudiante, esta asociación entre la creatividad y la psicopatología se daba en una u otra línea, dada la controversia encontrada en los estudios que la han investigado. Nuestra hipótesis es que la creatividad se relacionará de forma negativa con los síntomas psicopatológicos. 


\section{Método}

\section{Participantes}

La muestra seleccionada estuvo compuesta por 113 estudiantes de la Universidad de Murcia de diferentes carreras.

De los 113 participantes de la muestra recogida, el porcentaje de mujeres $(63,72 \%)$ fue mayor que el de hombres (36,28\%). La edad media de la muestra fue de 22 años. Los resultados pueden encontrarse en la tabla 1 . El 77,7 \% de los participantes informaron de un nivel socioeconómico medio, un 18,4\% un nivel bajo y un 3,9\% de un nivel alto. En la tabla 1 se pueden comprobar el perfil de la muestra.

Tabla 1. Datos descriptivos de la muestra en sexo y edad.

\begin{tabular}{lcc}
\hline & $\mathrm{N}(\%)$ & Edad media (d.t.) \\
\hline Hombres & $41(36,28)$ & $23,76(7,33)$ \\
Mujeres & $72(63,72)$ & $22,06(4,50)$ \\
Total & 113 & $22,65(5,68)$ \\
\hline
\end{tabular}

\section{Instrumentos}

LOT-R (Life Orientation Test Revised): el optimismo disposicional se ha evaluado con la medida Scheier, Carver y Bridges (1994) versión española de Ferrando, Chico y Tous (2002). Es un autoinforme que evalúa las expectativas generalizadas hacia resultados positivos o negativos sobre el futuro de manera que altas puntuaciones en este cuestionario indican alto optimismo disposicional, y bajas informan sobre bajo optimismo disposicional. Está compuesto por 10 ítems, 4 de los cuales son de relleno (ítems $2,5,6,8$ ) y no tiene validez para el análisis. Los 6 ítems restantes, 3 se puntúan de forma directa y los otros de indirecta. Se pregunta a los sujetos que indiquen el grado de acuerdo o desacuerdo con afirmaciones como "en tiempos difíciles, generalmente espero lo mejor" usando una escala de 5 puntos, donde 0 (muy en desacuerdo) y 4 (muy de acuerdo).

Los estudios psicométricos realizados con el inventario evidencian que su consistencia interna ( $\alpha$ de Cronbach) oscila entre .74 (Schou, Ekeber, Ruland, Sandwick y Karesen, 2004) 
y .78 (Scheier et al., 1994). En cuanto a las propiedades psicométricas de la versión española, Ferrando y colaboradores (2002) confirman una adecuada validez del cuestionario. Respecto a su fiabilidad Martínez, Reyes del Paso, García y González (2006) obtienen un $\alpha$ de .75.

CREA: para evaluar la creatividad se utilizó el test de Corbalán (CREA) que provee de una medida cuantitativa global de la creatividad. Este test requiere 4 minutos de duración para formular preguntas sobre una lámina que se presenta a los sujetos. El número de preguntas que generen se interpreta como un indicador de flexibilidad cognitiva y como una medida de la capacidad del sujeto para relacionar esquemas cognitivos algo muy relacionado con la creatividad (Corbalan, Martínez, Donolo, Tejerina y Limiñana, 2003). El instrumento ha sido empleado en otras ocasiones y ha sido galardonado por sus buenas cualidades psicométricas (premio TEA EDICIONES 2001). Ha mostrado estar relacionado con otros instrumentos y medidas de la creatividad representando entre ellos correlaciones elevadas. Según los datos el instrumento presento un coeficiente de fiabilidad de 0.7919 , indicando con ello buenas propiedades psicométricas.

SCL-90 (Symptom checklist 90 Revised): El cuestionario de 90 síntomas de Derogatis (1973) es un instrumento autoadministrado que evalúa la presencia e intensidad de 90 síntomas psicopatológicos psiquiátricos y psicosomáticos. La versión española fue desarrollada por Gónzalez de Rivera y colaboradores (1989) que encuentran unas adecuadas características psicométricas del instrumento. Sin embargo, no debe emplearse para el diagnóstico de psicopatológica (Thompson, 1989). Tras su aplicación se obtienen 9 dimensiones sintomáticas de psicopatología y tres índices globales de malestar. La duración de la prueba oscila entre 12 y 15 minutos. El ámbito de aplicación es de los 13 años en adelante. Las escalas comprenden síntomas relacionados con somatización (SOM), obsesión-compulsión (OBS), Sensibilidad interpersonal (timidez, vergüenza, sentirse inferior que los demás, hipersensibilidad a las opiniones de los demás)(INT), Depresión (DEP), ansiedad (ANS), hostilidad (HOS), ansiedad fóbica (FOB), ideación paranoide (PAR), psicoticismo (PSI) y una escala adicional de varios ítems no encuadrados en los anteriores (ADI). Cada item del SCL-90 se valora con una escala Likert de 5 puntos indicando el malestar peribido durante los últimos 7 días.

En cuanto a las propiedades psicométricas de la versión española, González de Rivera, De Las Cuevas, Rodriguez y Rodriguez (2002) confirman una adecuada validez del cuestio- 
nario. Respecto a su fiabilidad obtienen un $\alpha$ por encima de 0.81 en todas las escalas del SCL90.

Procedimiento y análisis estadístico

Se aplicaron las tres pruebas a una muestra de 113 estudiantes de universidad (72 mujeres y 41 hombres) en diferentes cursos y carreras aprovechando un curso de hablar en público que ofrecía la universidad. Se administraron las pruebas de creatividad, optimismo y síntomas psicopatológicos descritas.

Las puntuaciones obtenidas tras la corrección de los instrumentos se analizaron mediante el índice de correlación de pearson para evaluar el grado de asociación entre estas tres variables. Se tomó como estadísticamente significativo una $\mathrm{p}<0,05$. Asimismo, se realizaron análisis de regresión para evaluar el porcentaje de varianza de los síntomas psicopatológicos explicado por la variabe optimismo y creatividad. Tal como han hecho otros estudios para estudiar los niveles de creatividad y su relación con la psicopatología, se realizó además un análisis de varianza para la comparación de medias entre distintos niveles de creatividad y los síntomas psicopatológicos. Para llevar este análisis a cabo se procedió a agrupar a los sujetos en tres grupos (baja, media y alta creatividad) confeccionados a partir de puntos de corte obtenidos una puntuación típica por encima y por debajo de la media y se compararon las escalas de psicopatología del SCL-90 en función de esta agrupación.

\section{Resultados}

Diferencias de género en psicopatologia, creatividad y optimismo disposicional.

No se encontraron diferencias significativas de género en las variables de creatividad, optimismo disposicional y sintomas psicopatológicos. Tan solo se encuentran diferencias marginalmente significativas $(\mathrm{t}=1,879 ; \mathrm{p}=.063)$ en sintomatología depresiva indicando que las mujeres presentas más sintomatología depresiva que los hombres. Para una mayor información se puede consultar la Tabla 2. 
Tabla 2. Diferencias de medias entre sexo. Descriptivos (media y desviación típica) para cada sexo en las distintas dimensiones del SCL-90.

\begin{tabular}{|c|c|c|c|c|c|c|c|c|}
\hline & Género & $\mathrm{n}$ & Media & $\mathrm{Dt}$ & Error & $\mathrm{T}$ & g.1. & $\mathrm{P}$ \\
\hline \multirow{2}{*}{ SOM } & & & & ,74261 & & \multirow{2}{*}{1,602} & \multirow{2}{*}{108} & \multirow{2}{*}{.112} \\
\hline & Hom & 39 & 9 & 53921 & .08634 & & & \\
\hline \multirow{2}{*}{ OBS } & & 71 & & & & \multirow{2}{*}{1,038} & \multirow{2}{*}{108} & \multirow{2}{*}{.302} \\
\hline & Hom & 39 & 1,1231 & 67647 & .10832 & & & \\
\hline \multirow{2}{*}{ INT } & Muj & 71 & 34 & 2487 & .09789 & \multirow{2}{*}{1,131} & \multirow{2}{*}{108} & \multirow{2}{*}{.260} \\
\hline & Hom & 39 & & ,75582 & .12103 & & & \\
\hline \multirow{2}{*}{ DEP } & $\mathrm{Muj}$ & 71 & & 467 & & \multirow{2}{*}{1,879} & \multirow{2}{*}{108} & \multirow{2}{*}{.063} \\
\hline & Homl & 39 &, 8600 & 3306 & .10137 & & & \\
\hline \multirow{2}{*}{ ANS } & & 71 & & & & \multirow[b]{2}{*}{,6 } & \multirow{2}{*}{108} & \multirow{2}{*}{.518} \\
\hline & Hom & 39 & ,8872 & ,62668 & .100 & & & \\
\hline \multirow{2}{*}{ HOS } & & 71 & & 7100 & .07 & \multirow{2}{*}{,408 } & \multirow{2}{*}{108} & \multirow{2}{*}{.684} \\
\hline & Hon & 39 & ,74 & 8278 & .10 & & & \\
\hline \multirow{2}{*}{ FOB } & & 7 & & & & \multirow{2}{*}{,937 } & \multirow{2}{*}{108} & \multirow{2}{*}{.351} \\
\hline & Hom & 39 & ,3846 & 42499 & .068 & & & \\
\hline \multirow{2}{*}{ PAR } & & 71 & 1,4038 & 4519 & .112 & \multirow{2}{*}{,997 } & \multirow{2}{*}{108} & \\
\hline & & 3 & & & & & & .321 \\
\hline & & 71 & & & 22 & & & \\
\hline IS & & 39 & & & & & 100 & \\
\hline & & 71 & ,98 & ,72182 & .08 & & & \\
\hline IVIS & & 3 & ,908 & ,65669 & & , & 108 & 00 \\
\hline & & 71 & ,9962 & ,59245 & .07031 & & & \\
\hline & & 39 & ,8573 &, 52245 & .08366 & & & \\
\hline & & 71 & 46,4930 & 18,67074 & 2.21581 & 689 & 108 & 49 \\
\hline PS 1 & Homl & 39 & & & 3.25596 & (009, & 100 & .492 \\
\hline & & 71 & 1,8184 &, 52656 & .06249 & & & \\
\hline & Hombre & 39 & 1,6571 & ,45103 & .07222 & & 100 & 109 \\
\hline
\end{tabular}

Análisis correlacional

Se encontraron correlaciones negativas significativas entre optimismo y todos los aspectos psicopatológicos evaluados por el SCL-90 excepto hostilidad (HOS). La correlación más intensa fue entre depresión y optimismo $(r=-.502 ; p<.001)$ y entre dificultades interpersonales y optimismo $(r=-.447 ; p<.001)$. El índice de sufrimiento (GSI) también se relacionó inversamente con el optimismo medido a través del LOT-R $(r=-.459 ; p<.001)$. Estos resultados indican una relación inversa entre presentar síntomas psicopatológicos y los niveles de optimismo.

Las correlaciones entre los síntomas psicopatológicos y la creatividad fueron nulas. La relación entre la creatividad y el optimismo disposicional, aunque positiva, fue no significati- 
va y cercana a 0 ( $r=.035$; n.s.), indicando que son dos constructos diferentes y poco relacionados entre sí. En la Tabla 3 se muestra la correlación entre las escalas de síntomas psicopatológicos y su relación con el optimismo (LOT-R) y la creatividad (CREA).

Tabla 3. Correlaciones entre creatividad, optimismo y variables psicopatológicas medidas con el SCL-90 (* $p<0,05 ; * * p<0,01 ; * * *<0,001)$.

\begin{tabular}{ccc}
\hline & Lot-R & CREA \\
\hline SOM & $-.289^{* *}$ & .121 \\
OBS & $-.438^{* * *}$ & .089 \\
INT & $-.447 * * *$ & .026 \\
DEP & $-.502 * * *$ & .007 \\
ANS & $-.406 * * *$ & .106 \\
HOS & -.106 & .097 \\
FOB & $-.383 * * *$ & .013 \\
PAR & $-.401 * * *$ & .156 \\
PSI & $-.403 * * *$ & .043 \\
MIS & $-.273 * * *$ & .106 \\
GSI & $-.459 * * *$ & .089 \\
PST & $-.390^{* * *}$ & .073 \\
PSDI & $-.403 * * *$ & .076 \\
\hline
\end{tabular}

Análisis de regresión

Dado que las correlaciones podrían entenderse en dos sentidos (p.ej. puede ser que los que tienen menos psicopatología presentan más optimismo) se decidió hacer análisis de regresión para poder acercarse más a la consideración de una relación causal.

Los análisis de regresión efectuados utilizando la variable optimismo muestran que los síntomas psicopatológicos medidos a través del SCL-90 son predichos por la puntuación del LOT-R. Las variables mejor explicadas por el optimismo fueron los síntomas depresivos (DEP; $24,5 \%$ de varianza explicada) y el nivel general de sufrimiento somático y psicológico (GSI; 20,3\%). El resto de resultados se puede encontrar en la tabla 4. 
Tabla 4. Análisis de regresión simple utilizando la variable optimismo como predictor de cada una de las escalas de síntomas psicopatológicos del SCL-90.

\begin{tabular}{lllllll} 
LOT-R & $\mathbf{R}$ & $\mathbf{R}^{\mathbf{2}}$ & $\mathbf{R}^{\mathbf{2}}$ cor. & \multicolumn{2}{c}{ E. tip. Estimac. $\mathbf{F}$} & SIG. \\
\hline SOM & .289 & .084 & .075 & .62089 & 9.688 & .002 \\
OBS & .438 & .192 & .184 & .61029 & 25.152 & .000 \\
INT & .447 & .199 & .192 & .71382 & 26.416 & .000 \\
DEP & .502 & .252 & .245 & .61091 & 35.780 & .000 \\
ANS & .406 & .165 & .157 & .57877 & 20.957 & .000 \\
HOS & .106 & .011 & .002 & .66708 & 1.208 & .274 \\
FOB & .383 & .147 & .139 & .48079 & 18.221 & .000 \\
PAR & .401 & .161 & .153 & .86077 & 20.323 & .000 \\
PSI & .403 & .163 & .155 & .54352 & 20.582 & .000 \\
MIS & .273 & .074 & .066 & .066 & 8.530 & .004 \\
GSI & .459 & .211 & .203 & .50338 & 28.306 & .000 \\
PST & .390 & .152 & .144 & 17.72113 & 19.033 & .000 \\
PSDI & .403 & .162 & .154 & .45224 & 20.540 & .000
\end{tabular}

SOM = Somatización; OBS = Obsesión-Compulsión; INT = Sensibilidad Interpersonal; DEP $=$ Depresión; ANS $=$ Ansiedad; HOS $=$ Hostilidad; FOB = Ansiedad Fóbica; PAR = Ideación Paranoide; PSI = Psicoticismo; MIS = Miscelanea; PSDI = Índice de Distrés de Síntomas Positivos; GSI = Índice Global de Gravedad; PST =Total de Síntomas Positivos.

Cuando se realizaron los análisis de regresión utilizando la variable creatividad como variable predictora, se observó que no se encontró ninguna predicción significativa entre creatividad y las diversas dimensiones de psicopatología. En la tabla 5 se puede comprobar los resultados de estos análisis.

Tabla 5. Análisis de regresión simple utilizando la variable creatividad como predictor de cada una de las escalas de síntomas psicopatológicos del SCL-90.

\begin{tabular}{|c|c|c|c|c|c|c|}
\hline CREA & $\mathbf{R}$ & $\mathbf{R}^{2}$ & $\mathbf{R}^{2}$ cor. & E. tip. Estimac. F & & \\
\hline$\overline{\mathrm{SOM}}$ & .121 & .015 & .005 & .67411 & 1.550 & .216 \\
\hline OBS & .089 & .008 & -.002 & .68353 & .833 & .363 \\
\hline INT & .026 & .001 & -.009 & .80660 & .068 & .794 \\
\hline DEP & .007 & .000 & -.009 & .74056 & .005 & .943 \\
\hline ANS & .106 & .011 & .002 & .62733 & 1.202 & .275 \\
\hline HOS & .097 & .009 & .000 & .67003 & .999 & .320 \\
\hline FOB & .013 & .000 & -.009 & .50458 & .019 & .891 \\
\hline PAR & .156 & .024 & .015 & .92043 & 2.609 & .109 \\
\hline PSI & .043 & .002 & -.008 & .60282 & .196 & .659 \\
\hline MIS & .106 & .011 & .002 & .70129 & 1.194 & .277 \\
\hline GSI & .089 & .008 & -.001 & .57119 & .844 & .360 \\
\hline PST & .073 & .005 & -.004 & 19,21800 & .569 & .452 \\
\hline PSDI & .076 & .006 & -.004 & .50960 & .605 & .438 \\
\hline
\end{tabular}


Diferencia de medias en psicopatología entre distintos niveles de creatividad.

Se decidió estudiar las diferencias entre distintos niveles de creatividad (baja, media y alta), utilizando como puntos de corte una desviación típica por encima y por debajo de la media, con respecto a los síntomas de psicopatología. Se encontraron niveles de psicopatología diferentes en función de los niveles de creatividad. Para el grupo de menor creatividad aparecieron diferencias marginalmente significativas en las variables de ansiedad fóbica $(\mathrm{F}=$ $2.567 ; \mathrm{p}=.082)$ y la variable psicoticismo $(\mathrm{F}=3.384 ; \mathrm{p}=.038)$. Mientras que para el grupo de alta creatividad en ideación paranoide $(\mathrm{F}=3.052 ; \mathrm{p}=.052)$. Por lo general los participantes con niveles medios en creatividad presentan menores puntuaciones de psicopatología en la mayoría de escalas. Los resultados pueden encontrarse en la tabla 6.

Tabla 6. Diferencias de medias en psicopatología según nivel de creatividad. Media y desviación típica en cada grupo de creatividad (ANOVA)

\begin{tabular}{|c|c|c|c|c|c|c|c|c|}
\hline & $\begin{array}{l}\text { Niveles de } \\
\text { creatividad }\end{array}$ & $\mathbf{N}$ & Media & Dt & Error & g.l. & $\mathbf{F}$ & $\mathbf{P}$ \\
\hline \multirow{3}{*}{ SOM } & C. baja & 11 & ,9015 & ,62674 & , 18897 & & \multirow{4}{*}{,294 } & \multirow{4}{*}{.746} \\
\hline & C. media & 82 & ,9045 & ,68320 & ,07545 & 2 & & \\
\hline & C. alta & 14 & 1,0536 & ,70226 & 18769 & 104 & & \\
\hline \multirow{5}{*}{ OBS } & Total & 107 & ,9237 & 67585 & ,06534 & 106 & & \\
\hline & C. baja & 11 & 1,4545 & ,82263 & ,24803 & & \multirow{4}{*}{1,350} & \multirow{4}{*}{.264} \\
\hline & C. media & 82 & 1,1634 & 63877 & 07054 & 2 & & \\
\hline & C. alta & 14 & 1,3857 & 80274 & 21454 & 104 & & \\
\hline & Total & 107 & 1,2224 & 68299 & 06603 & 106 & & \\
\hline \multirow{4}{*}{ INT } & C. baja & 11 & 1,5051 & 1,13133 & ,34111 & & \multirow{4}{*}{1,784} & \multirow{4}{*}{.173} \\
\hline & C. media & 82 & 1,0759 &, 71261 & 07869 & 2 & & \\
\hline & C. alta & 14 & 1,3254 & 96714 & ,25848 & 104 & & \\
\hline & Total & 107 & 1,1526 & ,80305 & 07763 & 106 & & \\
\hline \multirow{5}{*}{ DEP } & C. baja & 11 & 1,3986 & ,89365 & ,26944 & & \multirow{4}{*}{1,715} & \multirow{4}{*}{185} \\
\hline & C. media & 82 & ,9728 & 66791 & 07376 & 2 & & \\
\hline & C. alta & 14 & 1,1044 & 94578 & 25277 & 104 & & \\
\hline & Total & 107 & 1,0338 & 73708 & ,07126 & 106 & & \\
\hline & C. baja & 11 & 1,1364 & ,82616 & 24910 & & \multirow{4}{*}{1,989} & \multirow{4}{*}{.142} \\
\hline \multirow{3}{*}{ ANS } & C. media & 82 &, 8732 & ,55534 &, 06133 & 2 & & \\
\hline & C. alta & 14 & 1,1714 & 80616 & 21545 & 104 & & \\
\hline & Total & 107 & ,9393 & 62793 & 06070 & 106 & & \\
\hline \multirow{5}{*}{ HOS } & C. baja & 11 & ,7424 & 64275 & 19380 & & \multirow{4}{*}{, 557} & \multirow{4}{*}{.575} \\
\hline & C. media & 82 & ,7642 & 64786 & 07154 & 2 & & \\
\hline & C. alta & 14 & 9643 & 82994 & 22181 & 104 & & \\
\hline & Total & 107 & ,7882 & 67003 & ,06477 & 106 & & \\
\hline & C. baja & 11 & 6883 & 83009 & 25028 & & 2,567 & .082 \\
\hline
\end{tabular}




\begin{tabular}{|c|c|c|c|c|c|c|c|c|}
\hline \multirow{3}{*}{ FOB } & C. media & 82 & ,3885 & ,42785 &, 04725 & 2 & & \\
\hline & C. alta & 14 & ,6020 & ,53797 & , 14378 & 104 & & \\
\hline & Total & 107 & ,4473 & ,50224 & ,04855 & 106 & & \\
\hline \multirow{3}{*}{ PAR } & C. baja & 11 & 1,3636 & 1,13240 & ,34143 & & \multirow{4}{*}{3,052} & \multirow{4}{*}{.052} \\
\hline & C. media & 82 & 1,2317 & ,79942 & ,08828 & 2 & & \\
\hline & C. alta & 14 & 1,8810 & 1,28673 & ,34389 & 104 & & \\
\hline \multirow{5}{*}{ PSI } & Total & 107 & 1,3302 & ,92739 & ,08965 & 106 & & \\
\hline & C. baja & 11 & ,9727 & ,74712 & ,22526 & & \multirow{4}{*}{3,384} & \multirow{4}{*}{.038} \\
\hline & C. media & 82 &, 5683 & ,49861 & ,05506 & 2 & & \\
\hline & C. alta & 14 &, 8643 & ,88457 & ,23641 & 104 & & \\
\hline & Total & 107 & ,6486 & 60053 & ,05806 & 106 & & \\
\hline \multirow{4}{*}{ MIS } & C. baja & 11 & 1,0519 & ,70933 & ,21387 & & \multirow{4}{*}{1,886} & \multirow{4}{*}{.157} \\
\hline & C. media & 82 &, 8955 & ,65563 & ,07240 & 2 & & \\
\hline & C. alta & 14 & 1,2755 & ,90084 & ,24076 & 104 & & \\
\hline & Total & 107 & ,9613 & ,70193 & ,06786 & 106 & & \\
\hline \multirow{4}{*}{ GSI } & C. baja & 11 & 1,1444 & ,74356 & 22419 & & \multirow{4}{*}{1,956} & \multirow{4}{*}{.147} \\
\hline & C. media & 82 &, 8911 & ,50976 & ,05629 & 2 & & \\
\hline & C. alta & 14 & 1,1484 & ,71808 & ,19192 & 104 & & \\
\hline & Total & 107 & ,9508 & ,57077 & ,05518 & 106 & & \\
\hline \multirow{4}{*}{ PST } & C. baja & 11 & 50,2727 & 20,89541 & 6,30020 & & \multirow{4}{*}{, 721} & \multirow{4}{*}{.489} \\
\hline & C. media & 82 & 44,4390 & 18,18838 & 2,00857 & 2 & & \\
\hline & C. alta & 14 & 49,2143 & 23,68764 & 6,33079 & 104 & & \\
\hline & Total & 107 & 45,6636 & 19,17887 & 1,85409 & 106 & & \\
\hline \multirow{4}{*}{ PSDI } & C. baja & 11 & 1,9095 & ,58382 & ,17603 & & \multirow{4}{*}{2,118} & \multirow{4}{*}{.125} \\
\hline & C. media & 82 & 1,7112 & ,48558 &, 05362 & 2 & & \\
\hline & C. alta & 14 & 1,9731 &, 54384 & , 14535 & 104 & & \\
\hline & Total & 107 & 1,7658 & ,50865 & ,04917 & 106 & & \\
\hline
\end{tabular}

\section{Discusión}

En este estudio se pretende comprobar si tanto el optimismo como la creatividad son factores protectores de psicopatología. Respecto al optimismo, se comprobó que existe una gran cantidad de investigaciones que ponen de manifiesto el efecto protector del optmimo, tanto en variables psicopatológicas como respecto a la salud física, en ambas concepciones del optimismo (como expectativa de futuro o como estilo explicativo). Los resultados de este estudio encontraron apoyo a la hipótesis propuesta del optimismo disposicional como factor protector de psicopatología, en todas las dimensiones del SCL-90 (a excepción de la dimensión de hostilidad). Los datos tienen una fuerte significación estadística, especialmente en la sintomatología depresiva, coincidiendo con la propuesta de Seligman (1998; 2005), en cuanto que propone un programa de fomento del optimismo para la prevención de la depresión en escuelas e institutos. Los datos de este estudio apuntaron en este sentido, proponiendo igual- 
mente, la necesidad de programas de promoción de bienestar psicológico y de prevención de psicopatología, que contengan el fomento del optimismo en los estudiantes. Es de resaltar también la fuerte relación negativa entre optimismo disposicional y dificultades interpersonales, por lo que el fomento del optimismo podría tener un efecto amplificador en programas que intentan mejorar las habilidades sociales y ser un factor protector de las dificultades interpersonales que puedan presentarse en la vida, teniendo en cuenta la gran importancia para el bienestar psicológico del apoyo social, que dependerá de tener unas buenas habilidades interpersonales (Inglés, 2007) y donde el optimismo también parece jugar un papel determinante (Seligman, 2005). Los análisis de regresión que se realizaron ayudan a poder acercarse más a hablar de una relación causal entre optimismo disposicional y psicopatología más alla de las correlaciones encontradas. Normalmente se han realizado programas de fomento del optimismo trabajando el estilo explicativo, principalmente ante situaciones negativas. Sin embargo, también se ha encontrado relación del estilo explicativo optimista ante situaciones positivas como factor protector ante la sintomatología depresiva (Sánchez y Méndez, 2009b) y su relación de este con el optimismo disposicional, por lo que el hecho de esperar buenos resultados en la vida junto con explicar estos buenos resultados mediante causas internas, estables y globales, podrían incrementar sustancialmente el carácter beneficioso sobre la salud y el bienestar psicológico que estas variables pudieran tener por separado (Sanjuán y Magallanes, 2006).

Respecto a la creatividad, aunque en este estudio no se encontró una relación significativa entre creatividad y psicopatología, el hallazgo de diferentes perfiles psicopatológicos encontrados en los distintos niveles de creatividad analizados, matiza la visión tradicional que apunta a una relación directa entre psicopatología y los grandes creativos (Eysenck, 1993). Los datos plantean un efecto en $U$ donde a ambos extremos de la creatividad se encuentran mayores nieveles de psicopatología. En los niveles bajos de creatividad encontramos mayores niveles de psicopatología en depresión, síntomas fóbicos y la escala de psicoticismo, lo que podría explicarse por mayor rigidez cognitiva. Sin embargo, el mayor nivel de ideación paranoide se encuentra en los participantes con un nivel alto de creatividad. Otros autores, analizando individuos creativos encontraron que los individuos creativos puntuaron más alto en desviación psicopática y esquizofrenia utilizando el MMPI, lo que fue interpretado como uso de pensamientos inusuales, menor inhibición y una mayor libertad imaginativa (MacKinon, 1962). La creatividad podría verse favorecida por estilos cognitivos que fomentan pensamientos alejados de lo habitual que, al igual que en estudio de MacKinon podría ser confundido con alejamiento de la realidad o síntomas psicóticos. Sin embargo, en el caso de una baja 
creatividad la falta de flexibilidad cognitiva también implicaría dificultades de adaptación que podría traducirse en síntomas psicopatológicos si el sujeto no puede dar respuesta a las demandas del medio.

En la presente investigación se encontraron datos que indicaban mayores puntuaciones en ideación paranoide en sujetos con alta creatividad. Una explicación posible para unas puntuaciones más elevadas en ideación paranoide (IP) puede hallarse en el hecho de que dos ítems que computan para obtener la puntuación en esta escala son "tener ideas o pensamientos que los demás no entienden" y "sentir que los demás no me valoran como merezco". En este sentido, el hecho de tener asociaciones lejanas y originales, descrito como requisito para la creatividad podría estar relacionado con estas experiencias.

En esta misma línea, mayores niveles de producción divergente también se han relacionado con niveles más elevados de asociaciones inusuales y menores acceso a sensaciones físicas que según la teoría de los marcadores somáticos de Damasio (Damasio A., 1994) se ha relacionado con mayores dificultades de condicionamiento y aprendizaje y subsiguientemente menores niveles de socialización lo que, según algunos autores, podría estar relacionado con mayores niveles de psicoticismo tal como fue teorizado por Eysenck (Galang, 2010). Esta situación podría explicar porque a niveles elevados de creatividad no encontramos un mayor efecto protector como a niveles medios: los efectos negativos de las asociaciones inusuales podrían también producir mayor psicopatología.

Resulta interesante destacar que los datos recogidos apuntan en la misma dirección que otro estudio en el que el grupo formado por los individuos con niveles medios de psicopatología presentaba mayores puntuaciones de creatividad, indicando una leve asociación con la psicopatología (Ghadirian, Gregoire y Kosmidis, 2001), coincidiendo parcialmente con las tendencias encontradas en este estudio.

Los datos encontrados aquí, sin embargo, contradicen otros estudios en los que la creatividad no solo no está asociada con la psicopatología sino que se encuentra que un predominio de emociones positivas beneficiaría a la creatividad (Lyubomirsky, King y Diener, 2005) pudiendo entroncarse con lo que otros autores han encontrado en relación con estados hipomaníacos facilitadores de la producción divergente (Nettle, 2002). 
Chavez-Eakle y colaboradores (2006), utilizando el test de Torrance, se encontraron relaciones inversas entre la creatividad y los síntomas psicopatológicos medidos a través del SCL-90 (Chavez-Eakle, Lara y Cruz-Fuentes, 2006). Los datos encontrados en este estudio no coinciden totalmente con el de Chavez-Eake ya que diferentes niveles de creatividad presentan diferentes síntomas psicopatológicos. Por otro lado, en el estudio de Chavez-Eakle (2006) se encontró que la psicopatología estaba más asociada a la personalidad que a la creatividad. Tal vez en ese estudio se estén midiendo, aparte de la variable creatividad, también aspectos relacionados con variables de personalidad o tal vez la muestra que utilizó en aquel caso (sujetos altamente creativos, sujetos controles y pacientes psiquiátricos) presenten características psicológicas diferentes a nuestro grupo de estudiantes y las variables creatividad y psicopatología se comporten de manera distinta. Sería conveniente considerar si el efecto beneficioso de la creatividad sobre los síntomas psicopatológicos podría estar mediado por otras variables como podría ser la inteligencia emocional. Olatoye y colaboradores (2010) encuentran que la creatividad se relaciona con la inteligencia emocional, siendo esta última asociada positivamente con la salud física y mental (Martínez, Piqueras y Ramos, 2010).

\section{Conclusiones}

Respecto al primer objetivo de este estudio se confirman los hallazgos de otras investigaciones que señalan el optimismo como factor protector de psicopatología (Carr, 2007; Ortiz et al., 2003; Séligman, 2005). En general se han encontrado resultados estadísticamente firmes que resaltan el efecto protector, principalmente en las dimensiones de depresión y dificultades interpersonales, en consonancia con aquellos programas de promoción del bienestar y de prevención de depresión basados principalmente en el fomento del optimismo y de las habilidades interpersonales desarrollados en contexto educativo (Seligman, 2005). Es de resaltar que se ha aportado nueva evidencia que confirma esta relación con los instrumentos de evaluación de optimismo disposicional, LOT-R (Scheier, Carver y Bridges, 1994) con la medida de psicopatología SCL-90 (Derogatis, Lipman y Covi, 1973), ya que no se encontraron otras investigaciones que lo hayan estudiado mediante estos dos instrumentos. Es importante señalar que los programas de fomento del optimismo se han centrado en trabajar el estilo explicativo, principalmente ante situaciones negativas. Sin embargo, también se ha encontrado relación entre el estilo explicativo optimista ante situaciones positivas como factor protector ante la sintomatología depresiva (Sánchez y Méndez, 2009b) y su relación de éste con el 
optimismo disposicional, por lo que incluir ambos en dichos programas aumentaría el bienestar psicológico que estas variables tendrían por separado (Sanjuán y Magallanes, 2006).

También se sugiere tener en cuenta nuevos conceptos y su relación con el optimismo, como el de flexibilidad explicativa y otros factores que pueden influir, como la autoestima, la percepción de controlabilidad sobre las situaciones estresantes o el grado de importancia otorgado a las mismas (Sánchez y Méndez, 2009b).

No se encontró, en este estudio, una relación significativa entre creatividad y psicopatología, aunque sí se hallaron datos que implican diferencias en perfiles psicopatológicos, en función de los niveles de creatividad. Si la creatividad puede ser independiente de la psicopatología y la creatividad se ve favorecida por las confluencias entre la creatividad y el optimismo pueden dar lugar a mejoras en la capacidad de elaborar productos creativos y en la mejora de la salud. Promoviendo estados emocionales que puedan favorecer la creatividad. Otros estudios han encontrado que la creatividad y las emociones positivas pueden estar asociadas y que deberían incluirse en los programas de prevención de la salud con el fin de incluirlos dentro de las variables de evaluación, especialmente en aquellos individuos que presenten mayor índice de psicopatología. Sin embargo, una mayor muestra, el empleo de otras medidas de psicopatología y creatividad y su relación con otras variables se requieren para dilucidar la relación entre los perfiles de creatividad y diferentes perfiles psicopatológicos.

Respondiendo a la pregunta que se formulaba en la introducción de este trabajo respecto si la creatividad supone un factor de protección ante ciertos tipos de psicopatología, los resultados de este estudio no encontraron una relación significativa entre ambas variables, aunque si se hallaron diferencias según el nivel de creatividad, resultando niveles medios de creatividad más relacionados con menos psicopatología, niveles bajos se relacionaron con mayores niveles de psicopatología en depresión, síntomas fóbicos y la escala de psicoticismo, ,mientras que para niveles altos su relación con una elevada distancia entre conceptos y asociaciones al límite del consenso y aceptación social podría resultar en una forma subclínica de psicopatología como sugiere Prentky (p. 262) (Glover, Ronning y Reinolds, 1989).

Por ello al considerar la relación de la creatividad con la psicopatología quizás se debe matizar, qué definicón de creatividad se esta barajando dependiendo del instrumento de medida utilizado, nivel de creatividad (bajo, medio o alto), tipo de psicopatología específica 
con la que se vincula y otras variables, como las diferencias de género, que diversos estudios (DeMoss, Milich, y DeMers, 1993; Andreasen 1987) han señalado que podrían ayudar a dilucidar esta compleja relación entre estas dos variables, lo que sin duda se presenta como un atractivo campo a seguir explorando.

Por otra parte poder integrar la novedad dentro de un contexto que pueda rescatar la innovación de forma productiva, amortiguando el efecto de la psicopatología es un reto para los individuos creativos en particular y en general para una sociedad que persiga el desarrollo y avance frente a las nuevas amenazas y oportunidades de este nuevo siglo.

\section{Referencias}

Abela, J. R. C. y Hankin, B. L. (2007). Handbook of depression in children and adolescents. Nueva York: Guilford Press.

Abramson L. Y., Seligman M. E. P. y Teasdale J. (1978). Learned helplessness in humans: Critique and reformulation. Journal of Abnormal Psychology, 87, 49-74.

Alonso-Monreal, C. (2000). Qué es la creatividad. Madrid: Biblioteca Nueva.

Andreasen, N. C. (1987). Creativity and mental illness: Prevalence rates in writers and their first-degree relatives. American Journal of Psychiatry, 144, 1288-1292.

Andreasen, N. C. (1996). Creativity and mental illness: A conceptual and historical overview. In J. J. Schildkraut \& A. Otero (Eds.), Depression and the spiritual in modern artHomage to Miro (pp. 2-14). New York: Wiley.

Avia, M. D. y Vázquez, C. (1998). Optimismo inteligente. Madrid: Alianza Editorial.

Baas, M., De Drew, C. K. W., Bernard A. N. (2008). A Meta-Analysis of 25 Years of MoodCreativity Research: Hedonic Tone, Activation, or Regulatory Focus? Psychological Bulletin, 134(6), 779-806.

Behrens, R.R. (1975). Lunatics, lovers and poets: On madness and creativity. Journal of Creative Behavior, 9, 228-232.

Cameron, N. (1947). The psychology of behavior disorders. Boston: Houghton Mifflin.

Carlsson, I. (2002). Anxiety and flexibility of defense related to high or low creativity. Creativity Research Journal, 14, 341-349.

Carr, A. (2007). Psicología positiva: La ciencia de la felicidad. Buenos Aires: Paidós.

Carver, C. y Gaines (1987). Optimism, pessimism, and postpartum depression. Cognitive therapy and Research, 11, 449-462. 
Carver, C. S. y Scheier, M. F. (1981). Attention and self-regulation: A control therapy approach to human behavior. New York: Springer Press.

Carver, C. S. y Scheier, M. F. (1998). On the self-regulation of behavior. New York: Cambridge University Press.

Casullo, M. M. (2004). SCL-90-R. Adaptación UBA. CONICET. Buenos Aires: Facultad de Psicología, Universidad de Buenos Aires.

Chavez-Eakle, R. A., Lara Ma del C. y Cruz-Fuentes, C. (2006). A Possible Bridge Between Creativity and Psychopathology. Educational Research, 18(1), p. 27-38.

Clapham, M. M. (2001). The effects of affect manipulation and information exposure on divergent thinking. Creativity Research Journal, 13, 335-350.

Claridge, G. y Blakey, S. (2009). Schizotypy and affective temperament: Relationships with divergent thinking and creativity styles. Personality and Individual Differences, 46(8), $820-826$.

Corbalán, F.J., Martínez, F., Donolo, D., Tejerina, M. y Limiñana, R.M. (2003). CREA Inteligencia Creativa. Una medidad cognitiva de la creatividad. Madrid: TEA Ediciones.

Cohen, J. (1988). Statistical power analysis for the behavioral sciences $\left(2 .{ }^{a}\right.$ ed). Hillsdale, Nueva Jersey: Erlbaum.

Csikszentmihalyi, M. (1998). El fluir y la psicología del descubrimiento y la invención. Barcelona: Paidós Transiciones.

Cutuli, J. J. (2004). Preventing externalizing symptoms and related features in adolescence. Unpublished honors thesis, University of Pennsylvania, Philadelphia, PA.

Cutuli, J.J., Chaplin, T.M., Gillham, J.E., Reivich, K.J. y Seligman, M.E.P. (2006). Preventing co-occurring depression symptoms in adolescents with conduct problems: The Penn Resiliency Program. New York Academy of Sciences, 1094, 282-286.

Damasio A., R. (1994). Descartes's Error: Emotion, Reason and the Human Brain. New York: Avon Books.

De Manzano, Ö., Cervenka, S., Karabanov, A., Farde, L. y UllÈn, F. (2010). Thinking Outside a Less Intact Box: Thalamic Dopamine D2 Receptor Densities Are Negatively Related to Psychometric Creativity in Healthy Individuals. PLoS ONE, 5(5), e10670.

DeMoss, Milich, y DeMers (1993). Gender, Creativity, Depression, and Attributional Style in Adolescents with High Academic Ability. Journal of Abnormal Child Psychology, 2 (4) $455-467$.

Derogatis, L.R., Lipman, R.S. y Covi, L. (1973). SCL-90. An outpatient psychiatric ratings cale. Preliminary report. Psychopharmacology Bulletin, 9, 13-27. 
Eysenck, H. J. (1993). Creativity and personality: suggestions for a theory. Psychological Inquiry, 4, 147-178.

Ferrando, P.J., Chico, E., y Tous, J.M. (2002). Propiedades psicométricas del test de optimismo Life Orientation Test. Psicothema, 14(3), 673-680.

Galang, A. J. R. (2010). The prosocial psychopath: Explaining the paradoxes of the creative personality. Neuroscience and Biobehavioural Reviews, 34, 1241-1248.

Ghadirian, A. M., Gregoire, P. y Kosmidis, H. (2001). Creativity and the Evolution of Psychopathologies. Creativity Research Journal. 13(2), 145-148.

Gillham, J. (2000). The science of optimism and hope. Filadelfia, PA: Templeton Foundation Press.

Gillham, J. E., Hamilton, J., Freres, D. R., Patton, K. y Gallop, R. (2006). Preventing depression among early adolescents in the primary care setting: A randomized controlled study of the Penn Resiliency Program. Journal of Abnormal Child Psychology. 34(2), 203-219.

Gillham, J. E., Jaycox, L. H., Reivich, K. J., Seligman, M. E. P. y Silver, T. (1990). The Penn Resiliency Program. (Also known as the Penn Depression Prevention Program and the Penn Optimism Program). Unpublished manuscript, University of Pennsylvania.

Glover, J., Ronning, R. y Reynolds, C. (1989). Handbook of creativity: Plenum Press.

González de Rivera, J.L., Derogatis, L.R., De las Cuevas, C., Gracia Marco, R., RodríguezPulido, F., Henry-Benítez, M. y Monterrey, A. L. The spanish version of the SCL-90R. Normative data in the general population. Clinical Psychometric Research, Towson, 1989.

González de Ricera, J. L., De las Cuevas, C., Rodríguez, M. y Rodríguez, F. (2002). Cuestionario de síntomas SCL-90 de Derogatis, L. Adaptación española. Madrid: TEA.

Guilford, J. P. y Strom, R. D. (1978). Creatividad y Educación. España. Ediciones Paidós.

Horowitz, J. L. y Garber, J. (2006). The prevention of depressive symptoms in children and adolescents: A meta-analytic review. Journal of Consulting and Clinical Psychology, 74, 401-415.

Inglés, C. J. (2007) Programa PEHIA. Enseñanza de habilidades interpersonales para adolescentes. Editorial Pirámide. Colección Ojos Solares.

Joscelyn E., F., Aprajita Mohanty, Herrington, J. D., Koven, N. S., Miller, G. A. y Heller, W. (2004). Neuropsychological evidence for dimensional schizotypy: Implications for creativity and psychopathology. Journal of Research in Personality, 38, 24-31. 
Kim, K. (2005). Can only intelligent people be creative. Journal of Secondary Gifted Education, 16, 57-66.

Lombroso, C. (1891). The man of genius. Londres. Walter Scott.

López, A. F., Kasanzew, A. y López, M. B. (2007). Efectos terapéuticos positivos a través del aumento del optimismo. Ciencias Psicológicas, I (2), 179-188.

Lubow, R. y Gewirtz, J. (1995). Latent inhibition in humans: Data, theory, and implications for schizophrenia. Psychological Bulletin, 117(1), 87-103.

Lyubomirsky, S., King, L. y Diener, E. (2005). The benefits of frequent positive affect: Does happiness lead to success? Psychological Bulletin, 131, 803-855.

MacKinon, D. W. (1962). The personality correlates of creativity: A study of American architects. Proceedings of the XIV International Congress of Aplied Psychology, Copenhagen. Ed. G. S. Nielsen. Vol. 2., 11-39. Copenhagen.

Martínez, A. E., Piqueras, J.A. y Ramos, V. (2010). Inteligencia Emocional en la Salud Física y Mental. Electronic Journal of Research in Educational Psychology, 8(2), 861-890.

Martínez, A., Reyes del Paso, G. A., García, A. y González, M. I. (2006). Optimismo/pesimismo disposicional y estrategias de afrontamiento del estrés. Psicothema, 18 (1), 66-72.

Maslow, A. H. (2005). La personalidad creadora. Editorial Kairós.

Mikulincer, M., Kedem, P. y Paz, D. (1990). Anxiety and categorization: I. The structure and boundaries of mental categories. Personality and Individual Differences, 11, 805814.

Nettle, D. (2002). Strong imagination: Madness, creativity and human nature. Oxford: Oxford University Press.

Nettle, D. (2006). Schizotypy and mental health amongst poets, visual artists, and mathematicians. Journal of Research in Personality, 40(6), 876-890.

Nolen-Hoeksema, S., Girgus, J.S. y Seligman, M.E.P. (1992). Predictors and consequences of childhood depressive symptoms: a 5-year longitudinal study. Journal of Abnormal Psychology, 101, 405-422.

Olatoye, R. A., Akintunde, S.O. y Yakasi, M. I. (2010). Inteligencia emocional, creatividad y logro académico en los estudiantes de empresariales. Electronic Journal of Research in Educational Psychology, 8(2), 763-786.

Ortiz, J., Ramos, N. y Vera-Villarroel, P. E. (2003). Optimismo y Salud: estado actual e implicaciones para la Psicología Clínica y de la Salud. Suma Psicológica, 10, 119-134. 
Otero, J., Luengo, A., Romero, E., Gómez, J. y Castro, C. (1998). Psicología de la personalidad. Manual de prácticas. Barcelona: Ariel, S.A.

Preckel, F., Holling, H. y Wiese, M. (2006). Relationship of intelligence and creativity in gifted and non-gifted students: An investigation of threshold theory. Personality and Individual Differences, 40(1), 159-170.

Richards, R. (2001). Creativity and the schizophrenia spectrum: More and more interesting. Creativity Research Journal, 13(1), 111-132.

Sánchez, O. y Méndez, F. X. (2009a). Prevención de la depresión infantil mediante el fomento del optimismo. Revista de Psicoterapia, 18, 77-89.

Sánchez, O. y Méndez, F. X. (2009b). El optimismo como factor protector de la depresión infantil y adolescente. Clínica y Salud, 20(3), 273-280.

Sánchez, O., Méndez, F. X. y Garber, J. (2009). La promoción de la curiosidad de los estudiantes para participar en un programa preventivo. Anuario de Psicología Clínica y de la Salud, 5, 29-39.

Sanjuán, P. y Magallanes, A. (2006). La relación entre optimismo disposicional y estilo atribucional y su capacidad predictiva en un diseño longitudinal. Revista de Psicología General y Aplicada, 59, 71-89

Scheier, M. y Carver, C. (1985). Optimism, coping and health: Assessment and implications of generalized outcome expectancies. Health Psychology, 4, 219-247.

Scheier, M. y Carver, C. (1987). Dispositional optimism and Physical well-being: the influence of generalized outcome expectancies on health. Journal of Personality, 55, 169210.

Scheier, M., Weintraub, J. K. y Carver, C. S. (1986). Coping whit stress: Divergent strategies of optimists and pessimists. Journal of Personality and Social Psychology, 51, 1257-1264.

Scheier, M.F., Carver, C.S., y Bridges, M.W. (1994). Distinguishing Optimism from neuroticism (and trait anxiety, self-mastery, and self-esteem): A reevaluation of the Life Orientation Test. Journal of Personality and Social Psychology, 67 (6), 1063-1078.

Schou, I., Ekeberg, O., Ruland, C.M., Sandwik, L. y Karesen, R. (2004).Pessimism as a predictor of emotional morbidity one year following breast cancer surgery. Psychooncology, 13, 309-320.

Seligman, M. E. P. (1998). Aprenda optimismo: Haga de la vida una experiencia maravillosa. Barcelona: Grijalbo.

Seligman, M. E. P. (2005). La Auténtica Felicidad. Ediciones B. 
Seligman, M. E. P. y Csikszentmihalyi, M. (2000). Positive psychology: An introduction. American Psychologist, 55, 5-14.

Seligman, M. E. P., Reivich, K., Jaycox, L. y Gillham, J. (2005). Niños optimistas. Barcelona: Random House Mondadori, S.A.

Seligman, M.E.P., Peterson, C., Kaslow, N. J., Tanenbaum, R.J., Alloy, L.B. y Abramson, L.Y. (1984). Attributional style and depressive symptoms among children. Journal of Abormal Psychology, 83(2), 235-238.

Stoneham, A. C. S. y Coughtrey, A. E. (2009). The role of schizotypy and creativity in a group problem-solving task. Personality and Individual Differences, 46, 827-831.

Thompson, C. (1989). Anxiety, en The instruments of Psychiatry Research. Ed. Chris Thompson.

Torres Jiménez, A., Robert, A., Tejero, A., Boget, T., y Pérez de los Cobos, J. (2006) Indefensión aprendida y dependencia de sustancias. Trastornos Adictivos, 8, 168-175.

Vandervert, L. R., Schimpf, P.H. y Liu, H. (2007). How working memory and the cerebellum collaborate to produce creativity and innovation. Creativity Research Journal, 19, 118.

Vera, B. (2008). Psicología positiva. Una nueva forma de entender la psicología. Calamar Ediciones.

Volkow, N. D., Fowler, J. S., Wang, G. J. y Swanson, J. M. (2004). Dopamine in drug abuse and addiction: results from imaging studies and treatment implications. Molecular Psychiatry, 9, 557-569.

Woody, E. y Claridge, G. (1977). Psychoticism and thinking. British Journal of Social and Clinical Psychology, 16(3), 241-248. 